\title{
Two Weight Characterization of New Maximal Operators
}

\section{Hu Yunpeng, Cao Yonghui}

Department of Mathematics and Systems Sciences, Xinjiang University, Urumqi, China

\section{Email address:}

husousuke@163.com (Hu Yunpeng),13579402215@163.com (Cao Yonghui)

\section{To cite this article:}

Hu Yunpeng, Cao Yonghui. Two Weight Characterization of New Maximal Operators. Pure and Applied Mathematics Journal. Vol. 8, No. 3, 2019, pp. 47-53. doi: 10.11648/j.pamj.20190803.11

Received: June 23, 2019; Accepted: July 19, 2019; Published: August 5, 2019

\begin{abstract}
For the last twenty years, there has been a great deal of interest in the theory of two weight. In the present paper, we investigate the two weight norm inequalities for fractional new maximal operator on the Lebesgue space. More specifically, we obtain that the sufficient and necessary conditions for strong and weak type two weight norm inequalities for a new fractional maximal operators by introducing a class of new two weight functions. In the discussion of strong type two weight norm inequalities, we make full use of the properties of dyadic cubes and truncation operators, and utilize the space decomposition technique which space is decomposed into disjoint unions. In contrast, weak type two weight norm inequalities are more complex. We have the aid of some good properties of $A_{p}$ weight functions and ingeniously use the characteristic function. What should be stressed is that the new two weight functions we introduced contains the classical two weights and our results generalize known results before. In this paper, it is worth noting that $w(\mathrm{x}) d x$ may not be a doubling measure if our new weight functions $\omega \in A_{p}(\varphi)$.. Since $\varphi(|Q|) \geq 1$, our new weight functions are including the classical Muckenhoupt weights.
\end{abstract}

Keywords: Two Weight, Maximal Operator, Lebesgue Space

\section{Introduction}

In 1972, Muckenhopt [1] established the $A_{p}$ weight theory when studying the Lebesgue boundedness of classical Hardy-Littlewood maximal operators. Subsequently, he further obtained the two weight boundedness of Hardy-Littlewood maximal operators and founded that the $A_{p}$ two weight condition is a necessary and sufficient condition for the two weight weak boundedness of Hardy-Littlewood maximal operators. In addition, he discussed strong boundedness. Muckenhopt and Wheeden [2] founded that the $A_{p}$ two weight condition is only a necessary but not sufficient condition for Hardy-Littlewood maximal operator and Hilbert transform to have two weight strong boundedness, which is essentially different from the one weight case. Therefore, as a generalization of the one weight case, it is more difficult to discuss the boundedness of operators with two weights than with one weight. In 1982, great progress was made in the two weighted results. Sawyer [3] obtained the necessary and sufficient conditions for the two weight $(u, v)$ of Hardy-Littlewood maximal operators to be bounded from $L^{p}(v d x)$ to $L^{p}(u d x)$ with $1<p \leq \infty$. In 2000 , Cruz-Uribe [4] gave a new proof of this result, and we can see a lot of work related to this topic in this paper, for example, see [5-8].
Next, some necessary definitions and notations are given. In this paper, $Q(x, r)$ denotes the cube centered at $x$ and of the sidelength $t$. Similarly, given $Q=Q(x, r)$ and $\lambda>0$, we will write $\lambda Q$ for the $\lambda$-dilate cube, which is the cube with the same center $x$ and with sidelength $\lambda t$. $C$ denote constants independent of parameters and may take different values in different places. Let $E \in R^{n}, \chi_{E}$ denote the characteristic function of $E .|E|$ denote the Lebesgue measure of $E$. $p^{\prime}$ denote the conjugate index of $p$, i.e. $1 / p+1 / p^{\prime}=1$.

Definition $1.1^{[9]}$. Let $\varphi(t)=(1+t)^{\alpha_{0}}, t \geq 0, \alpha_{0} \geq 0$. $\omega \geq 0$ a.e. and $\omega \in L_{l o c}^{p}\left(R^{n}\right)$.

(i) If there is a constant $C$ such that for all cubes $Q=$ $Q(x, r)$ with radius $r$ centered on $x$,

$$
\left(\frac{1}{\varphi(|Q|)|Q|} \int_{Q} \omega(y) d y\right)\left(\frac{1}{\varphi(|Q|)|Q|} \int_{Q} \omega^{-\frac{1}{p-1}}(y) d y\right)^{p-1} \leq C,
$$

Then we say that $\omega \in A_{p}(\varphi)(p>1)$.

(ii) If there is a constant $C$ such that

$$
M_{\varphi}(\omega)(x) \leq C \omega(x), \text { a.e. } x \in R^{n},
$$

where, 


$$
M_{\varphi} f(x)=\sup _{x \in Q} \frac{1}{\varphi(|Q|)|Q|} \int_{Q}|f(y)| d y
$$

Then we say that $\omega \in A_{1}(\varphi)$.

The classical Hardy-Littlewood maximal operator $M$ is defined by

$$
M f(x)=\sup _{x \in Q} \frac{1}{|Q|} \int_{Q}|f(y)| d y .
$$

Obviously, $f(x) \leq M_{\varphi} f(x) \leq M f(x)$, a.e. $x \in R^{n}$ and $M_{\varphi} f(x)$ is lower semi-continuous.

Since $\varphi(|Q|) \geq 1$, so $A_{p}\left(R^{n}\right) \subset A_{p}(\varphi)$ for $1<p<\infty$, where $A_{p}\left(R^{n}\right)$ denote the classical Muckenhoupt weights; see [10]. It is well know that if $\omega \in A_{\infty}\left(R^{n}\right)=\cup_{p \geq 1} A_{p}\left(R^{n}\right)$, then $\omega(x) d x$ be a doubling measure, i.e. there exist a constant $C>0$ for any cube $Q$ such that

$$
\omega(2 Q) \leq C \omega(Q) .
$$

But Tang pointed out in [9] that if $\omega \in A_{p}(\varphi)$, then $\omega(x) d x$ may not be a doubling measure. In fact, let $0 \leq \gamma \leq$ $n \alpha_{0}$, It is easy to check that $\omega(x)=(1+|x|)^{-(n+\gamma)} \notin$ $A_{\infty}\left(R^{n}\right)$ and $\omega(x) d x$ are not a double measures, but $\omega(x)=(1+|x|)^{-(n+\gamma)} \in A_{1}(\varphi)$.

The new maximal operators were firstly introduced by Tang [9] in connection with weighted $L^{p}$ inequalities for pseudo-diffferential operators with smooth symbols and their commutators by using a class of new weight functions which include Muckenhoupt weight functions. It can control a class of important operators, such as pseudo-difffferential operator

$$
T f(x):=\int_{R^{n}} \sigma(x, \xi) e^{2 \pi i x \cdot \xi} \hat{f}(\xi) d \xi,
$$

where $f$ is a Schwartz function and $\hat{f}$ denotes the Fourier transform of $f$, Symbol $\sigma(x, \xi) \in S_{1, \delta}^{0}$ with $0<\delta \leq 1$. In particular, A. Laptev [11] proved that any $S_{1,0}^{0}$ pseudo-diffferential operator is a standard Calderón-Zygmund operator; see also [12-15]. Applying the new maximal functions, Pan [16] obtained the strong type and weak end-point estimates for certain classes of multilinear operators and their iterated commutators with new BMO functions.

In this paper, the fractional form of a new maximal operator considered by Tang in [9] is introduced: given $0 \leq \alpha<1$, the fractional new maximal function $M_{\varphi}^{\alpha} f(x)$ is defined as

$$
M_{\varphi}^{\alpha} f(x):=\sup _{x \in Q} \frac{1}{(\varphi(|Q|)|Q|)^{1-\alpha}} \int_{Q}|f(y)| d y,
$$

where the supremum is taken over all the cube containing $x$.

Next, we introduce a new class of two weight functions, which includes the classical two weights in [3].

Definition 1.2. Let $1 \leq p \leq q<\infty, 0 \leq \alpha<1$ and $u, v \in L_{\text {loc }}^{1}\left(R^{n}\right)$. If for every cube $Q$,

(i) When $1<\mathrm{p}<\infty$,

$$
\frac{1}{(\varphi(|Q|)|Q|)^{1-\alpha}}\left(\int_{Q} u(x) d x\right)^{\frac{1}{q}}\left(\int_{Q} v(x)^{1-p^{\prime}} d x\right)^{\frac{1}{p^{\prime}}} \leq C .
$$

(ii) When $\mathrm{p}=1$, for a. e. $\mathrm{x} \in \mathrm{Q}$,

$$
\frac{1}{(\varphi(|Q|)|Q|)^{1-\alpha}}\left(\int_{Q} u(x) d x\right)^{\frac{1}{q}} \leq C v(x) .
$$

Then we say that $(u, v) \in A_{p, q}^{\alpha}(\varphi)$. It is easy to know that $A_{p, q}^{\alpha}\left(R^{n}\right) \subset A_{p, q}^{\alpha}(\varphi)$, with $A_{p, q}^{\alpha}\left(R^{n}\right)$ denote classical two weight.

We end this section with the outline of this paper. Section 2 contains Theorem 2.1.1, Theorem 2.2.1 and the proofs of them. we extend the corresponding strong and weak results to the fractional new maximal operators. In Section 3, we give a conclusion.

\section{Method and Results}

\subsection{Strong Boundedness of New Maximal Operators}

The purpose of this paper is to study the strong and weak type inequalities of fractional new maximal operators by introducing a new class of two weight functions containing the classical two weight, and obtain their two weight characterization in Lebesgue spaces.

The main result of this section is to obtain the two weight strong boundedness of fractional new maximal operators.

Theorem 2.1.1. Let $1<p \leq q<\infty, 0 \leq \alpha<1$ and $(u, v) \in A_{p, q}^{\alpha}(\varphi)$. Then the following statements are equivalent:

(i) For every cube $Q$,

$$
\begin{aligned}
& \left(\int_{Q}\left(M_{\varphi}^{\alpha}\left(v^{1-p^{\prime}} \chi_{Q}\right)(x)\right)^{q} u(x) d x\right)^{1 / q} \\
& \leq C\left(\int_{Q} v(x)^{1-p^{\prime}} d x\right)^{1 / p}
\end{aligned}
$$

(ii) For every $f \in L^{p}(v)$,

$\left(\int_{R^{n}}\left(M_{\varphi}^{\alpha} f(x)\right)^{q} u(x) d x\right)^{1 / q} \leq C\left(\int_{R^{n}}|f(x)|^{p} v(x) d x\right)^{1 / p}$.

The following lemma is needed to prove the theorem.

Lemma 2.1.2. Let $0 \leq \alpha<1, f \geq 0$ is a locally integrable function. If for every cube $Q$ and some $t>0$ such that

$$
\frac{1}{(\varphi(|Q|)|Q|)^{1-\alpha}} \int_{Q}|f(y)| d y>t,
$$

Then there exists a dyadic cube $P$ such that $Q \subset 3 P$ and 


$$
\frac{1}{(\varphi(|P|)|P|)^{1-\alpha}} \int_{P} f(y) d y>2^{\alpha-1-n} t .
$$

Proof. Take $k \in Z$ makes $2^{k-1} \leq \ell(Q)<2^{k}$, so there exists dyadic cubes $P_{1}, P_{2}, \ldots, P_{N}, 1 \leq N \leq 2^{n}$ whose generate by $2^{k}$ and intersect $Q$. Since $\ell\left(P_{j}\right)=2^{k}>l(Q)$, by Vitali covering lemma, we get $Q \subset 3 P_{j}$ for every $j$. In addition, it is assumed that there is no dyadic cube $P$ such that

$$
\int_{P} f(y) d y>\frac{t(\varphi(|Q|)|Q|)^{1-\alpha}}{2^{n}}
$$

Then,

$$
\int_{Q} f(y) d y \leq \sum_{j=1}^{N} \int_{P_{j}} f(y) d y \leq \sum_{j=1}^{N} \frac{t(\varphi(|Q|)|Q|)^{1-\alpha}}{2^{n}} \leq t(\varphi(|Q|)|Q|)^{1-\alpha} .
$$

This contradicts the hypothesis. Therefore, there exists a dyadic cube $P$,

$$
\frac{1}{(\varphi(|P|)|P|)^{1-\alpha}} \int_{P} f(y) d y>\frac{t(\varphi(|Q|)|Q|)^{1-\alpha}}{2^{n}(\varphi(|P|)|P|)^{1-\alpha}} \geq 2^{\alpha-1-n} t .
$$

Next, we give the proof of the theorem.

Proof of Theorem 2.1.1. Set $\sigma(x)=v(x)^{1-p^{\prime}}$. In (ii), take $f=\sigma \chi_{Q}$, it is easy to see that (ii) implicature (i). Next, we prove that (i) implicature (ii). Firstly, without losing generality, Let $f \in L^{p}(v)$ be a nonnegative bounded function with compact support. This ensures that $M_{\varphi}^{\alpha} f(x)$ is almost everywhere finite. $R^{n}$ is decomposed in the following way:

$$
R^{n}=\bigcup_{k \in Z} \Omega_{k}, \Omega_{k}=\left\{x \in R^{n}: 2^{k}<M_{\varphi}^{\alpha} f(x) \leq 2^{k+1}\right\} .
$$

Then, for each $k$ and $x \in \Omega_{k}$, there exists a cube $Q_{x}^{k}$ containing $x$ such that

$$
\frac{1}{\left(\varphi\left(\left|Q_{x}^{k}\right|\right)\left|Q_{x}^{k}\right|\right)^{1-\alpha}} \int_{Q_{x}^{k}} f(y) d y>2^{k},
$$

Therefore, by Lemma 2.1.2, there exists a dyadic cube $P_{x}^{k}$ such that $Q_{x}^{k} \subset 3 P_{x}^{k}$ and

$$
\begin{gathered}
\frac{1}{\left(\varphi\left(\left|P_{x}^{k}\right|\right)\left|P_{x}^{k}\right|\right)} \int_{P_{x}^{k}} f(y) d y>2^{\alpha-1-n} 2^{k} . \quad \text { (1) } \begin{array}{l}
\{(j, k) \in \mathbb{N} \quad \mathbb{Z}:|k| \leq K\} \text {. Since } P_{j}^{k} \\
E_{j}^{k} \subset \Omega_{k}, \text { we get }
\end{array} \\
I_{k}=\int_{\cup_{k=-K}^{K} \Omega_{k}}\left(M_{\varphi}^{\alpha} f(x)\right)^{q} u(x) d x=\sum_{(j, k) \in \Lambda_{K}} \int_{E_{j}^{k}}\left(M_{\varphi}^{\alpha} f(x)\right)^{q} u(x) d x \\
\leq \sum_{(j, k) \in \Lambda_{K}} u\left(E_{j}^{k}\right)\left(2^{k+1}\right)^{q} \leq C \sum_{(j, k) \in \Lambda_{K}} u\left(E_{j}^{k}\right)\left(\frac{1}{\left(\varphi\left(\left|P_{j}^{k}\right|\right)\left|P_{j}^{k}\right|\right)^{1-\alpha}} \int_{P_{j}^{k}} f(y) d y\right)^{q} \\
=C \sum_{(j, k) \in \Lambda_{K}} u\left(E_{j}^{k}\right)\left(\frac{1}{\left(\varphi\left(\left|3 P_{j}^{k}\right|\right)\left|3 P_{j}^{k}\right|\right)^{1-\alpha}} \int_{3 P_{j}^{k}} \sigma(y) d y\right)^{q}\left(\frac{\int_{P_{j}^{k}}\left(f \sigma^{-1}\right)(y) \sigma(y) d y}{\int_{3 P_{j}^{k}} \sigma(y) d y}\right)^{q} \\
=\mathrm{C} \int_{\mathcal{Y}} \mathrm{T}_{\mathrm{k}}\left(\mathrm{f} \sigma^{-1}\right)^{\mathrm{q}} \mathrm{dv},
\end{gathered}
$$

This estimate shows that for each fixed $k$, the dyadic cube $P_{x}^{k}$ is bounded. Therefore, there exists such a succollection of disjoint dyadic cubes $\left\{P_{j}^{k}\right\}_{j}$ that for a $j$, each $Q_{x}^{k}$ is contained in $3 P_{j}^{k}$. Therefore, $\Omega_{k} \subset \cup_{j} 3 P_{j}^{k}$. Next, we decompose the $\Omega_{k}$ :

$$
\begin{aligned}
E_{1}^{k}=3 P_{1}^{k} \cap \Omega_{k}, E_{2}^{k} & =\left(3 P_{2}^{k} \backslash 3 P_{1}^{k}\right) \cap \Omega_{k}, \ldots, E_{j}^{k} \\
& =\left(3 P_{j}^{k} \backslash \bigcup_{r=1}^{j-1} 3 P_{r}^{k}\right) \cap \Omega_{k}, \ldots
\end{aligned}
$$

So we get

$$
R^{n}=\bigcup_{k=Z} \Omega_{k}=\bigcup_{j, k} E_{j}^{k}
$$

where $E_{j}^{k}$ 's are pairwise disjont for all $j$ and $k$. Fix a sufficiently large constant $K>0$ and Set $\Lambda_{K}=$ $\{(j, k) \in \mathbb{N} \quad \mathbb{Z}:|k| \leq K\}$. Since $P_{j}^{k}$ satisfies (1), using

where $\mathcal{Y}=\mathbb{N} \quad \mathbb{Z}, \mathrm{v}$ is a measure in $\mathcal{Y}$, with

$$
v(j, k)=u\left(E_{j}^{k}\right)\left(\frac{1}{\left(\varphi\left(\left|3 P_{j}^{k}\right|\right)\left|3 P_{j}^{k}\right|\right)^{1-\alpha}} \int_{3 P_{j}^{k}} \sigma(y) d y\right)^{q}, \quad T_{k} h(j, k)=\frac{\int_{P_{j}^{k}} h(y) \sigma(y) d y}{\int_{3 P_{j}^{k}} \sigma(y) d y} \chi_{\Lambda_{K}(j, k)} \text {. }
$$

For each measurable function $h$, operator $T_{k}$ is defined by In this case, if we can be proved that $T_{k}: L^{p}\left(R^{n}, \sigma\right) \rightarrow$ 
$L^{q}(\mathcal{Y}, v)$ is uniformly bounded, then it can be obtained from (2),

$$
\begin{gathered}
I_{k} \leq C \int_{y} T_{k}\left(f \sigma^{-1}\right)^{q} d v \leq C\left(\int_{R^{n}}\left(f \sigma^{-1}\right)^{p} \sigma d x\right)^{\frac{q}{p}} \\
=C\left(\int_{R^{n}} f^{p} v d x\right)^{\frac{q}{p}} .
\end{gathered}
$$

consistency of $I_{k}$ and the control convergence theorem. Therefore, only proving that $T_{k}: L^{p}\left(R^{n}, \sigma\right) \rightarrow L^{q}(\mathcal{Y}, v)$ uniformly bounded is necessary. $T_{k}: L^{\infty}\left(R^{n}, \sigma\right) \rightarrow L^{\infty}(\mathcal{Y}, v)$ is obvious, by Marcinewicz interpolation theorem, it is only to prove the uniform boundedness of $T_{k}: L^{1}\left(R^{n}, \sigma\right) \rightarrow$ $L^{q / p, \infty}(\mathcal{Y}, v)$. So we only need to prove that

The expected inequalities can be obtained by using the

$$
v\left\{(j, k) \in \mathcal{Y}: T_{k} h(j, k)>\lambda\right\} \leq C\left(\frac{1}{\lambda} \int_{R^{n}}|h(x)| \sigma(x) d x\right)^{q / p}, \lambda>0 .
$$

For this reason, fix $h \geq 0$ is a bounded function with compact support. Set

$$
F_{\lambda}=\left\{(j, k) \in \mathcal{Y}: T_{k} h(j, k)>\lambda\right\}=\left\{(j, k) \in \Lambda_{K}: T_{k} h(j, k)>\lambda\right\} .
$$

Since $E_{j}^{k} \subset 3 P_{j}^{k}$, we have

$$
\begin{gathered}
v\left(F_{\lambda}\right)=\sum_{(j, k) \in F_{\lambda}} u\left(E_{j}^{k}\right)\left(\frac{1}{\left(\varphi\left(\left|3 P_{j}^{k}\right|\right)\left|3 P_{j}^{k}\right|\right)^{1-\alpha}} \int_{3 P_{j}^{k}} \sigma(y) d y\right)^{q} \\
\leq \sum_{(j, k) \in F_{\lambda}} \int_{E_{j}^{k}}\left(\frac{1}{\left(\varphi\left(\left|3 P_{j}^{k}\right|\right)\left|3 P_{j}^{k}\right|\right)^{1-\alpha}} \int_{3 P_{j}^{k}} \sigma(y) d y\right)^{q} u(x) d x \\
\leq \sum_{(j, k) \in F_{\lambda}} \int_{E_{j}^{k}}\left(M_{\varphi}^{\alpha}\left(\sigma \chi_{3 P_{j}^{k}}\right)(x)\right)^{q} u(x) d x .
\end{gathered}
$$

The dydic cube in the family $\left\{P_{j}^{k}:(j, k) \in F_{\lambda}\right\}$ is bounded. In fact, if $(j, k) \in F_{\lambda}$, then $|k| \leq K$, and for each $k$, the cube $\left\{P_{j}^{k}\right\}_{j}$ is bounded. This allows us to select the largest subset family $\left\{P_{i}\right\}_{i}$ for each $(j, k) \in F_{\lambda}, P_{j}^{k} \subset P_{i}$. By $E_{j}^{k}$ 's are pairwise disjoint and $E_{j}^{k} \subset 3 P_{j}^{k}$,

$$
\begin{gathered}
v\left(F_{\lambda}\right) \leq \sum_{i} \sum_{P_{j}^{k} \subset P_{i}} \int_{E_{j}^{k}}\left(M_{\varphi}^{\alpha}\left(\sigma \chi_{3 P_{j}^{k}}\right)(x)\right)^{q} u(x) d x \\
\leq \sum_{i} \int_{3 P_{j}^{k}}\left(M_{\varphi}^{\alpha}\left(\sigma \chi_{3 P_{j}^{k}}\right)(x)\right)^{q} u(x) d x \\
\leq C \sum_{i}\left(\int_{3 P_{i}} \sigma(x) d x\right)^{q / p},
\end{gathered}
$$

where we used the definition of two weight. Since the cube $P_{i}$ is selected from the maximal disjoint subcollection $\left\{P_{j}^{k}:(j, k) \in F_{\lambda}\right\}$, there exists $P_{i}=P_{j}^{k}$ for each $i$, with $(j, k) \in F_{\lambda}$. In this case, $T_{k} h(j, k)>\lambda$, and since $(j, k) \in F_{\lambda}$,

$$
\begin{aligned}
\int_{3 P_{i}} \sigma(x) d x=\int_{3 P_{j}^{k}} \sigma(x) d x & \\
& <\frac{1}{\lambda} \int_{P_{j}^{k}} h(x) \sigma(x) d x=\frac{1}{\lambda} \int_{P_{i}} h(x) \sigma(x) d x .
\end{aligned}
$$

Notice that $\mathrm{q} / \mathrm{p} \geq 1$, the maxima of cube $P_{i}$ and their disjoints, we have

$$
\begin{gathered}
v\left(F_{\lambda}\right) \leq C \sum_{i}\left(\frac{1}{\lambda} \int_{P_{i}} h(x) \sigma(x) d x\right)^{q / p} \\
\leq C\left(\sum_{i} \frac{1}{\lambda} \int_{P_{i}} h(x) \sigma(x) d x\right)^{q / p} \\
\leq C\left(\frac{1}{\lambda} \int_{R^{n}} h(x) \sigma(x) d x\right)^{q / p},
\end{gathered}
$$

where $C$ is independent of $Q$, so the proof is completed.

\subsection{Weak Boundedness of New Maximal Operators}

The main result of this section is to obtain the two weight weak boundedness of fractional new maximal operators.

Theorem 2.2.1. Let $1 \leq p \leq q<\infty, 0 \leq \alpha<1$ and $(u, v) \in A_{p, q}^{\alpha}(\varphi)$. Then the following statements are equivalent:

(i) $(u, v) \in A_{p, q}^{\alpha}(\varphi)$;

(ii) $M_{\varphi}^{\alpha}: L^{p}(v) \rightarrow L^{q, \infty}(u)$, i.e. for any $\lambda>0$,

$u\left\{x \in R^{n}: M_{\varphi}^{\alpha} f(x)>\lambda\right\} \leq \frac{C}{\lambda^{q}}\left(\int_{R^{n}}|f(x)|^{p} v(x) d x\right)^{q / p} ;$ 
(iii) For every $f \geq 0$ and cube $Q$,

$$
\begin{aligned}
\left(\frac{1}{(\varphi(|Q|)|Q|)^{1-\alpha}}\right. & \left.\int_{Q} f(x) d x\right)^{q} u(Q) \\
& \leq C\left(\int_{Q} f(x)^{p} v(x) d x\right)^{q / p} .
\end{aligned}
$$

Proof of Theorem 2.2.1. The proof will be carried out in the following manner:

$$
\text { (ii) } \Rightarrow \text { (iii) } \Rightarrow \text { (i) } \Rightarrow \text { (iii) } \Rightarrow \text { (ii) }
$$

(ii) $\Rightarrow$ (iii) Take $f \geq 0$ and cube $Q$ such that

$$
\tilde{f}_{\alpha, Q}=\frac{1}{(\varphi(|Q|)|Q|)^{1-\alpha}} \int_{Q} f(x) d x>0 .
$$

If $0<\lambda<\tilde{f}_{\alpha, Q}$ and $x \in Q$, we have

$$
\left(\frac{1}{(\varphi(|Q|)|Q|)^{1-\alpha}} \int_{S} f(x) d x\right)^{q} u(Q) \leq C\left(\int_{S} f(x)^{p} v(x) d x\right)^{q / p} .
$$

If $f \equiv 1$, then

$$
\left(\frac{|S|}{(\varphi(|Q|)|Q|)^{1-\alpha}}\right)^{q} u(Q) \leq C v(S)^{q / p} .
$$

According to $[5,388$ pages $]$, we can only consider non-trivial cases and prove that $(u, v) \in A_{p, q}^{\alpha}(\varphi)$. Firstly, for $1<p<\infty$, take $f(x)=v(x)^{1-p^{\prime}}$. Fix $Q$, Set

$$
S_{j}=\left\{x \in Q: v(x)>\frac{1}{j}\right\}, j=1,2, \ldots
$$

Then $f$ is bounded on each $S_{j}$ and $\int_{S_{j}} v^{1-p^{\prime}} d x<\infty$. Using (3), with $S=S_{j}$ and $f=f \chi_{S}$, we get

$$
\begin{aligned}
\left(\frac{1}{(\varphi(|Q|)|Q|)^{1-\alpha}} \int_{S_{j}} v(x)^{1-p^{\prime}} d x\right)^{q} u(Q) \\
\leq C\left(\int_{S_{j}} v(x)^{1-p^{\prime}} d x\right)^{q / p} .
\end{aligned}
$$

Each integral is finite, so

$$
\frac{1}{(\varphi(|Q|)|Q|)^{1-\alpha}}\left(\int_{S_{j}} v(x)^{1-p^{\prime}} d x\right)^{q-\frac{q}{p}} u(Q) \leq C,
$$

i.e.

$\frac{1}{(\varphi(|Q|)|Q|)^{1-\alpha}}\left(\int_{Q} u(x) d x\right)^{\frac{1}{q}}\left(\int_{S_{j}} v(x)^{1-p^{\prime}} d x\right)^{\frac{1}{p^{\prime}}} \leq C$.

In addition, $S_{1} \subset S_{2} \subset \cdots$ and $\bigcup_{j} S_{j}=\{x \in Q: v(x)>0\}$. Let $j \rightarrow \infty$, we get $\lambda<\tilde{f}_{\alpha, Q}=\frac{1}{(\varphi(|Q|)|Q|)^{1-\alpha}} \int_{Q} f(x) \chi_{Q} d x \leq M_{\varphi}^{\alpha}\left(f \chi_{Q}\right)(x)$,

Then $Q \subset\left\{y \in R^{n}: M_{\varphi}^{\alpha}\left(f \chi_{Q}\right)(y)>\lambda\right\}$. By (ii),

$$
\begin{aligned}
u(Q) \leq u\left\{y \in R^{n}:\right. & \left.M_{\varphi}^{\alpha}\left(f \chi_{Q}\right)(y)>\lambda\right\} \\
& \leq \frac{C}{\lambda^{q}}\left(\int_{Q} f(x)^{p} v(x) d x\right)^{q / p},
\end{aligned}
$$

Thus,

$$
\left(\tilde{f}_{\alpha, Q}\right)^{q} u(Q) \leq C\left(\int_{Q} f(x)^{p} v(x) d x\right)^{q / p},
$$

Therefore, (iii) hold.

(iii) $\Rightarrow$ (i) For $p>1$, Let $f \geq 0$. For any $S \subset Q$, in (iii), take $f=f \chi_{S}$ to have

$\frac{1}{(\varphi(|Q|)|Q|)^{1-\alpha}}\left(\int_{Q} u(x) d x\right)^{\frac{1}{q}}\left(\int_{\{x \in Q: v(x)>0\}} v(x)^{1-p^{\prime}} d x\right)^{\frac{1}{p^{\prime}}}$ $\leq C$.

Therefore, $v>0$ a.e., $(u, v) \in A_{p, q}^{\alpha}(\varphi)$.

For $p=1$, notice that (4) can be written in the following form: for every $Q$, with $S \subset Q,|S|>0$,

$$
\frac{1}{(\varphi(|Q|)|Q|)^{1-\alpha}}\left(\int_{Q} u(x) d x\right)^{1 / q} \leq C \frac{v(S)}{|S|} .
$$

Fix $Q$, consider

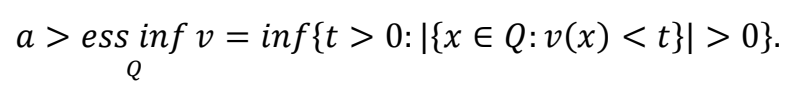

Set $S_{a}=\{x \in Q: v(x)<a\} \subset Q$. Then, $\left|S_{a}\right|>0$ and

$$
\begin{gathered}
\frac{1}{(\varphi(|Q|)|Q|)^{1-\alpha}}\left(\int_{Q} u(x) d x\right)^{1 / q} \leq \frac{C}{\left|S_{a}\right|} \int_{S_{a}} v(x) d x \\
\leq \frac{C}{\left|S_{a}\right|} a\left|S_{a}\right|=C a
\end{gathered}
$$

For each $a>$ ess $i n f_{Q} v$, the upper formula is hold, so

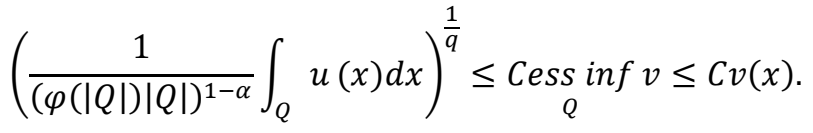

i.e. $(u, v) \in A_{1, q}^{\alpha}(\varphi)$.

(i) $\Rightarrow$ (iii) First, we consider the case of $p=1$. For $f \geq 0$ and every cube $Q$, 


$$
\begin{gathered}
\left(\frac{1}{(\varphi(|Q|)|Q|)^{1-\alpha}} \int_{Q} f(x) d x\right)^{q} u(Q)=\left(\int_{Q} f(x) \frac{1}{(\varphi(|Q|)|Q|)^{1-\alpha}}\left(\int_{Q} u(y) d y\right)^{\frac{1}{q}} d x\right)^{q} \\
\leq C\left(\int_{Q} f(x) v(x) d x\right)^{q} .
\end{gathered}
$$

The last inequality used $(u, v) \in A_{1, q}^{\alpha}(\varphi)$.

On the other hand, when $1<p<\infty$, the Hölder inequality has

$$
\begin{aligned}
& \left(\frac{1}{(\varphi(|Q|)|Q|)^{1-\alpha}} \int_{Q} f(x) d x\right)^{q}=\frac{1}{(\varphi(|Q|)|Q|)^{(1-\alpha) q}}\left(\int_{Q} f(x) v(x)^{\frac{1}{p}} v(x)^{-\frac{1}{p}} d x\right)^{q} \\
& \leq \frac{1}{(\varphi(|Q|)|Q|)^{(1-\alpha) q}}\left(\int_{Q} f(x)^{p} v(x) d x\right)^{q / p}\left(\int_{Q} v(x)^{1-p^{\prime}} d x\right)^{q / p^{\prime}}
\end{aligned}
$$

Since $(u, v) \in A_{p, q}^{\alpha}(\varphi)$,

$$
\begin{gathered}
\left(\frac{1}{(\varphi(|Q|)|Q|)^{1-\alpha}} \int_{Q} f(x) d x\right)^{q} u(Q) \\
\leq \frac{1}{(\varphi(|Q|)|Q|)^{(1-\alpha) q}}\left(\int_{Q} f(x)^{p} v(x) d x\right)^{q / p}\left(\int_{Q} v(x)^{1-p^{\prime}} d x\right)^{q / p^{\prime}} \int_{Q} u(x) d x \\
=\left(\int_{Q} f(x)^{p} v(x) d x\right)^{q / p}\left\{\frac{1}{(\varphi(|Q|)|Q|)^{1-\alpha}}\left(\int_{Q} u(x) d x\right)^{1 / q}\left(\int_{Q} v(x)^{1-p^{\prime}} d x\right)^{1 / p^{\prime}}\right\}^{q} \\
\leq C\left(\int_{Q} f(x)^{p} v(x) d x\right)^{q / p} .
\end{gathered}
$$

Therefore, (iii) is hold.

(iii) $\Rightarrow$ (ii) Let $f \in L_{l o c}^{p}\left(R^{n}\right), u(Q)>0$, then

$$
\begin{aligned}
\left(\frac{1}{(\varphi(|Q|)|Q|)^{1-\alpha}}\right. & \left.\int_{Q} f(x) d x\right)^{q} u(Q) \\
& \leq C\left(\int_{Q} f(x)^{p} v(x) d x\right)^{q / p}<\infty,
\end{aligned}
$$

$$
\begin{aligned}
\frac{1}{\left(\varphi\left(\left|r_{x}\right|^{n}\right)\left|r_{x}\right|^{n}\right)^{1-\alpha}} & \int_{Q\left(x, r_{x}\right)} f(y) d y \\
& >2^{\alpha-1}\left(\frac{1+2^{n}|Q|}{1+|Q|}\right)^{-\alpha_{0}} \lambda .
\end{aligned}
$$

In

particular, $\quad\left|r_{x}\right|^{n} \leq$

thus $f \in L_{l o c}^{1}\left(R^{n}\right)$. So it can be assumed that $f \in L^{1}\left(R^{n}\right)$, by defining $f_{k}=f \chi_{Q(0, k)}$, then we have $f_{k} \rightarrow f$. In this case, the $k$ limit of $f_{k}$ in (ii) is independent of each constant C, so (ii) about $f$ holds. With these, we will prove that the inequality of expectation holds when $f \geq 0, f \in L^{p}(v) \cap L^{1}\left(R^{n}\right)$.

Set

$$
E_{\lambda}=\left\{x \in R^{n}: M_{\varphi}^{\alpha} f(x)>\lambda\right\}
$$

If $x \in E_{\lambda}$, from the definition of $\varphi$ and the relationship between maximal function and central maximal function, there exists $r_{x}>0$ such that

$$
\begin{gathered}
u\left(E_{\lambda}\right) \leq \sum_{j} u\left(Q\left(x_{j}, 3 r_{j}\right)\right) \\
\leq C \sum_{j}\left(\frac{1}{\left(\varphi\left(\left|Q\left(x_{j}, 3 r_{j}\right)\right|\right)\left|Q\left(x_{j}, 3 r_{j}\right)\right|\right)^{1-\alpha}} \int_{Q\left(x_{j}, r_{j}\right)} f(x) d x\right)^{-q}\left(\int_{Q\left(x_{j}, r_{j}\right)} f(x)^{p} v(x) d x\right)^{\frac{q}{p}} \\
\leq C \sum_{j} \frac{1}{\left(\varphi\left(\left|r_{x}\right|^{n}\right)\left|r_{x}\right|^{n}\right)^{1-\alpha}}\left(\int_{Q\left(x_{j}, r_{j}\right)} f(x) d x\right)^{-q}\left(\int_{Q\left(x_{j}, r_{j}\right)} f(x)^{p} v(x) d x\right)^{\frac{q}{p}}
\end{gathered}
$$




$$
\begin{gathered}
\leq \frac{c}{\lambda^{q}}\left(\sum_{j} \int_{Q\left(x_{j}, r_{j}\right)} f(x)^{p} v(x) d x\right)^{\frac{q}{p}} \\
\leq \frac{c}{\lambda^{q}}\left(\int_{R^{n}} f(x)^{p} v(x) d x\right)^{\frac{q}{p}},
\end{gathered}
$$

where, we consider that $q / p \geq 1$ and $Q\left(x_{j}, r_{j}\right)$ are pairwise disjoint.

\section{Conclusion}

This paper gives the sufficient and necessary conditions for strong and weak type two weight norm inequalities for a new fractional maximal operators by introducing a class of new two weight functions which include classical two weight functions on Lebesgue spaces and our main results will shed some new lights on boundedness of other operators and their commutators on Lebesgue spaces. In addition, we can also consider the two weight characterization of new maximal operators on Morrey spaces.

\section{Acknowledgements}

The authors would like to thank the Referees and Editors for carefully reading the manuscript and making several useful suggestions.

This work is supported by the Natural Science Foundation of XinJiang Province (2016D01C044).

\section{References}

[1] B. Muckenhoupt. Weighted norm inequalities for the Hardy maximal function. Trans. Amer. Math. Soc.. 165, 207-226, 1972.

[2] B. Muckenhoupt, R. L. Wheeden. Two weight function norm inequalities for the Hardy-Littlewood maximal function and the Hilbert transform. Studia Math.. 55 (3): 279-294, 1976.

[3] E. T. Sawyer. A characterization of a two-weight norm inequality for maximal operators. Studia Math.. 75 (1), 1-11, 1982.

[4] D. Cruz-Uribe. New proofs of two-weight norm inequalities for the maximal operator. Georgian Math. J.. 7 (1), 33-42, 2000.
[5] E. T. Sawyer. Weighted norm inequalities for fractional maximal operators. Amer. Math. Soc., 1, 283-309, 1981.

[6] C. Perez. Two weighted inequalities for potential and fractional type maximal operators. Indiana Math. J., 43, 663-683, 1994.

[7] R. Wheeden. A characterization of some weighted norm inequalities for the fractional maximal function. Studia Math., 107, 257-272, 1993.

[8] A. Gogatishvili, V. Kokilashvili. Criteria of strong type two-weighted inequalities for fractional maximal functions. Georgian Math. J., 3, 423-446, 1996.

[9] L. Tang. Weighted norm inequalities for pseudo-differential oprators with smooth symbols and their commutators. J. Funct. Anal., 262 (4): 1603-1629, 2012.

[10] J. García-Cuerva, J. Rubio de Francia. Weighted norm inequalities and related topics. Amsterdam-New York, North-Holland, 387-395, 1985.

[11] A. Laptev. Spectral asympotics of a class of Fourier integral operators (Russian). Trudy Moskov. Mat. Obshch., 43, 92-115, 1981.

[12] J. Alverez, J. Hounie. Estimates for the kernel and continuity properties of pseudo diffferential operators. Ark. Mat. 28, 1-22, 1990.

[13] P. Auscher, M. Taylor. Paradifffferential operators and commutators estimates. Comm. Partial Diffferential Equations 20, 1743-1775, 1995.

[14] R. Coifman, C. Fefffferman. Weighted norm inequalities for maximal functions and singular integrals, Studia. Math. 51, 241-250, 1974.

[15] S. Chanillo, A. Torchinsky. Sharp fuction and weighted L estimates for a class of pseudo-difffferential operators. Ark. Mat. 28, 1-25, 1985.

[16] G. X. Pan, L. Tang. New weighted norm inequalities for certain classes of multilinear operators and their iterated commutators. Potential Analysis, 43 (3), 371-398, 2015. 